\title{
Role of transforming growth factor $\beta-1$ in the pathogenesis of pelvic organ prolapse: A potential therapeutic target
}

\author{
CHENG LIU $^{1 *}$, YING WANG $^{2 *}$, BING-SHU LI $^{1 *}$, QING YANG $^{1}$, JIAN-MING TANG $^{1}$, \\ JIE MIN ${ }^{1}$, SHA-SHA HONG ${ }^{1}$, WEN-JUN GUO ${ }^{1}$ and LI HONG $^{1}$ \\ ${ }^{1}$ Department of Gynecology and Obstetrics, Renmin Hospital of Wuhan University; \\ ${ }^{2}$ Department of Anesthesiology, The Central Hospital of Wuhan, Tongji Medical College, \\ Huazhong University of Science and Technology, Wuhan, Hubei 430060, P.R. China
}

Received February 12, 2017; Accepted June 13, 2017

DOI: $10.3892 /$ ijmm.2017.3042

\begin{abstract}
The present study aimed to reveal the metabolic alterations of the extracellular matrix (ECM) in uterosacral ligament (USL) with pelvic organ prolapse (POP) and to explore the role of transforming growth factor- $\beta 1$ (TGF- $\beta 1$ ) in pathogenesis of POP. For this purpse, 60 participants who underwent hysterectomy for benign indications were enrolled, 30 of which had symptomatic POP (grade II, III or IV) and composed the POP group, and the other 30 had asymptomatic POP (grade I or less) and served as the controls. Collagen fibers, elastin,matrix metalloproteinase (MMP)-2/9, tissue inhibitor of matrix metalloproteinases (TIMP)- 2 and TGF- $\beta 1$ were examined by Masson's trichrome staining, immunohistochemistry and RT-qPCR using USL biopsies. In vitro, human USL fibroblasts (hUSLFs) were primary cultured, pre-treated with recombinant TGF- $\beta 1(0,5$, or $10 \mathrm{ng} / \mathrm{ml})$ and then subjected to cyclic mechanical stretching (CMS; 0 or $5,333 \mu \varepsilon$ strain). Changes in the expression levels of collagen type I/III, elastin, TIMP-2, MMP-2/9 and Smad were detected. Our results revealed that at the tissue level, the expression of collagen fibers, elastin, TIMP-2 and TGF- $\beta 1$ was significantly reduced in the POP group, while the activities of MMP-2/9 were significantly
\end{abstract}

Correspondence to: Professor Li Hong, Department of Gynecology and Obstetrics, Renmin Hospital of Wuhan University, 99 Zhangzhidong Street, Wuhan, Hubei 430060, P.R. China

E-mail: 2013103020057@whu.edu.cn

*Contributed equally

Abbreviations: POP, pelvic organ prolapse; ECM, extracellular matrix; COL, collagen; PCR, polymerase chain reaction; IHC, immunohistochemistry; TGF, transforming growth factor; IOD, integrated optical density; TIMP, tissue inhibitor of matrix metalloproteinases; MMPs, matrix metalloproteinases; USL, uterosacral ligament; CMS, cyclic mechanical stretching; hUSLF, human uterosacral ligamental fibroblast; Smad, small mothers against decapentaplegic

Key words: pelvic organ prolapse, extracellular matrix, matrix metalloproteinase, tissue inhibitor of matrix metalloproteinases, transforming growth factor- $\beta 1$ upregulated, compared with the control group. Statistical analysis indicated that the mRNA expression of TGF- $\beta 1$ inversely correlated with the severity of POP partially. Our in vitro experimental data demonstrated that a CMS of $5333 \mu \varepsilon$ strain promoted the degradation of ECM proteins, inhibited the synthesis of TIMP-2, and upregulated the proteolytic activities of MMP-2/9. Pre-treatment with TGF- $\beta 1$ attenuated the loss of ECM by stimulating the synthesis of TIMP-2 and inhibiting the activities of MMP-2/9 through the TGF- $\beta 1 / \mathrm{Smad} 3$ signaling pathway. On the whole, our data indicate that the reduced anabolism and increased catabolism of ECM proteins in USL are the pathological characteristics of POP. TGF- $\beta 1$ not only has a specific value in predicting the severity of $\mathrm{POP}$, but should also be considered as a novel therapeutic target for POP.

\section{Introduction}

POP is a global health concern that is associated with increasing morbidity and economic burden, and it is estimated to affect almost $50 \%$ of women above 60 years of age (1). Approximately $11 \%$ of females require at least one surgery for prolapse in their lifetime (2), and the direct cost incurred is equivalent to billions of dollars annually in the United States (3). POP contributes to a low quality of life and has become one of the most common indications for gynecological surgery among post-menopausal women (4). The pathophysiological mechanisms of POP, however, have not yet been fully elucidated. The pelvic organs are mainly connected and supported by the levator ani muscle complex, the cardinal and uterosacral ligament (USL) and endopelvic fascia. The parauterine ligaments along with the endopelvic fascia compose a connective tissue network, supporting the maintenance of the normal position of the vagina and uterus. The disruption and dysfunction in this connective tissue network may lead to the weakening of support, and eventually, urogenital prolapse $(5,6)$.

The present study focused on the metabolic alterations in connective tissue in USL as it has been considered to be one of the most important apparatuses in the pelvic supporting network (7). USL is composed of vessels, nerves, smooth muscle and connective tissue, and collagen fibers constitute $70-80 \%$ of the connective tissue (8). Collagen and elastin are the main extracellular matrix (ECM) components in USL, which provide 
strength and flexibility. Collagen type I (COL1) strengthens the connective tissues, whereas collagen type III (COL3) and elastin contribute to flexibility (9). Precursor collagens (COL1A1 and COL3A1) and elastin are synthesized by fibroblasts, and are then secreted into the ECM as the raw material of fibril assembly $(6,22-24)$. The degradation of collagens depends on the activity of the interstitial matrix metalloproteinases (MMPs). MMPs are divided into subgroups according to substrate. MMP-1, -8 and -13 (collagenase) cleave pro-collagen fibers into fragments. The cleaved collagen fragments and elastin are then digested into amino acids by MMP-2/9 (gelatinases) (10). Tissue inhibitor of matrix metalloproteinases (TIMP) is the specific antagonist of MMPs (11). TGF- $\beta 1$, as an important regulator of fibrotic metabolism, and has been widely reported in the process of fibrosis and degenerative fibrotic disease $(6,12)$; however, the role of TGF- $\beta 1$ in USL with POP has not been reported to date, at least to the best of our knowledge. Based on the available data, we hypothesized that TGF- $\beta 1$ may play a regulatory role in POP.

The importance of ECM metabolism in USL has been well documented. However, the majority of the published data was established on the investigation of the vaginal wall $(13,14)$ and endopelvic fascia $(15,16)$; research data on USL are lacking. Additionally, there are many controversies regarding the trends in the alterations of the levels of collagen, elastin, MMPs and TIMP, which has led to uncertainty and is not beneficial for further study. In the present study, we aimed to identify the metabolic status the ECM proteins mentioned above in USL from women with POP, and to explore the exact role of TGF- $\beta 1$ in the pathogenesis of POP and the related mechanisms.

\section{Materials and methods}

Participants and specimen collection. Human USL biopsies were collected from patients after obtaining informed consent with the approval of the Ethics Committee of Renmin Hospital of Wuhan University, Wuhan, China. A total of 60 subjects were selected among female patients who underwent hysterectomy for benign indications during the period of March 2011 to June 2014 at the Department of Gynecology of Renmin Hospital of Wuhan University. Based on POP quantitative examination (17), 30 patients with POP stages II-IV who underwent hysterectomy as part of pelvic reconstruction surgery were included in the POP group. The other 30 subjects with non-POP or asymptomatic POP (POP grade I) who underwent hysterectomy for cervical intraepithelial neoplasia or dysfunctional uterine bleeding comprised the control group. All the included subjects were post-menopausal for at least 1 year. Women with malignant tumors, pelvic endometriosis and acute pelvic inflammation, as well as those undergoing hormone replacement therapy (HRT) were excluded from the study. The 2 groups were matched for age, menopause status, parity and body mass index (BMI). Biopsies were obtained from the left USL during hysterectomy. Specimens were prepared and preserved according to various protocols.

Masson's trichrome staining. The specimens were fixed with $4 \%$ paraformaldehyde, embedded in paraffin, cut into $4-\mu \mathrm{m}$-thick sections, and mounted onto coated slides. Masson's trichrome staining was performed to quantify collagen fibers using the HT15 kit (Sigma, St. Louis, MO, USA) according to the standard protocol.

Immunochemistry (IHC). The UltraSensitive SP kit-9710 (Maixin Biotech Co., Ltd., Fuzhou, China) was used for IHC. The slides were heated for $30 \mathrm{~min}$ at $60^{\circ} \mathrm{C}$, de-paraffinized and rehydrated in a graded alcohol series. Antigen retrieval was performed through boiling in citrate $(\mathrm{pH}<6)$ or EDTA buffer ( $\mathrm{pH}>9$ ), according to the product datasheets. This was followed by incubation with $3 \% \mathrm{H}_{2} \mathrm{O}_{2}$ for $10 \mathrm{~min}$ at room temperature to inactivate endogenous peroxidase, and blocking with 5\% goat serum for $15 \mathrm{~min}$, and incubationg with primary antibodies overnight at $4^{\circ} \mathrm{C}$. Following incubation with streptavidin peroxidase for $10 \mathrm{~min}$ at room temperature, secondary antibodies were added. Finally, immune reaction was visualized using the DAB-0031 kit (Maixin Biotech Co., Ltd.). The specimens were washed with phosphate-buffered saline (PBS) after each step in the protocol. The primary antibody was replaced with PBS for the negative controls.

The antibodies used were as follows: COL1A1 antibody (diluted at 1/100; BA0325; Boster Inc., Wuhan, China), COL3A1 antibody (diluted at 1/150; BA0326; Boster Inc.), elastin antibody (diluted at 1/100; ab23747; Abcam, Cambridge, UK), MMP-2 antibody (diluted at 1/75; BA3716; Boster Inc.), MMP-9 antibody (diluted at 1/100; BA0573; Boster Inc.), TIMP-2 antibody (diluted at 1/100; sc-5539; Santa Cruz Biotechnology, Inc., Santa Cruz, CA, USA) TGF- $\beta 1$ antibody (diluted at 1/100; ab92486; Abcam).

Immunoactivity was quantified with the IOD, integrated optical density (IOD) value and the mean density was captured using Image-Pro Plus version 6.0 software (Media Cybernetics, Inc., Rockville, MD, USA); mean density $=$ IOD/area.

Western blot analysis. Tissue samples were homogenized in RIPA buffer and quantified using the BCA Protein Assay kit (P0012; Beyotime Biotech, Jiangsu, China), prior to mixing with loading buffer. Following degeneration, the protein samples were separated in $10 \%$ SDS-PAGE and transferred onto PVDF membranes at $200 \mathrm{~mA}$ for $1-3 \mathrm{~h}$ at $4^{\circ} \mathrm{C}$. The blots were blocked with 5\% non-fat milk in TBS for $1 \mathrm{~h}$ at room temperature and incubated with primary antibody overnight at $4^{\circ} \mathrm{C}$. The PVDF membranes were washed with TBST thrice. Subsequently, the blots were incubated with IRDye $800 \mathrm{CW}$ goat anti-rabbit/mouse secondary antibodies (diluted at 1/10,000; LI-COR Inc., Lincoln, NE, USA) at room temperature for $1 \mathrm{~h}$. The Odyssey imaging system (LI-COR Inc.) was used for western blot analysis.

The antibodies used were as follows: elastin antibody (1:200 dilution; ab23747; Abcam), MMP-2 antibody (1:200 dilution, sc-53630), MMP-9 antibody (1:100 dilution, sc-13520), TIMP-2 antibody (1:200 dilution; sc-5539), COL1A1 antibody (1:400 dilution; sc-8784), COL3A1 antibody (1:400 dilution; sc-28888) (all from Santa Cruz Biotechnology, Inc.), TGF- $\beta 1$ antibody (1:250 dilution; ab92486; Abcam) and GAPDH antibody (1:1,000 dilution; sc-20357; Santa Cruz Biotechnology, Inc.), p-Smad3 (1:1,000 dilution, \#9520), Smad3 (1:1,000 dilution, \#9523), p-Smad2 (1:1,000 dilution, \#3108), Smad2 (1:1,000 dilution, \#5339), Smad4 (1:1,000 dilution, \#38454; all from Cell Signaling Technology, Inc., Danvers, MA, USA). 
Table I: Sequences of primers used for RT-qPCR.

\begin{tabular}{|c|c|c|c|}
\hline Primer & Accession no. & Sequence $\left(5^{\prime} \rightarrow 3^{\prime}\right)$ & Product length (bp) \\
\hline COL1A1 & NM_000088.3 & $\begin{array}{l}\text { Forward: CAAGACGAAGACATCCCACCAATC } \\
\text { Reverse: ACAGATCACGTCATCGCACAACA }\end{array}$ & 135 \\
\hline COL3A1 & NM_000090.3 & $\begin{array}{l}\text { Forward: TCGCTCTGCTTCATCCCACTAT } \\
\text { Reverse: CTTCCAGACATCTCTATCCGCAT }\end{array}$ & 101 \\
\hline Elastin & NM_006329.3 & $\begin{array}{l}\text { Forward: CGCTCTAGCATCCCTCCTCT } \\
\text { Reverse: GCAAGGTGGCTATTCCCAGT }\end{array}$ & 122 \\
\hline MMP-2 & NM_001127891.2 & $\begin{array}{l}\text { Forward: AGTTTCCATTCCGCTTCCAG } \\
\text { Reverse: CGGTCGTAGTCCTCAGTGGT }\end{array}$ & 100 \\
\hline MMP-9 & NM_004994.2 & $\begin{array}{l}\text { Forward: GTCCACCCTTGTGCTCTTCC } \\
\text { Reverse: GACTCTCCACGCATCTCTGC }\end{array}$ & 117 \\
\hline TIMP-2 & NM_003255.4 & $\begin{array}{l}\text { Forward: TCTGGAAACGACATTTATGG } \\
\text { Reverse: GTTGGAGGCCTGCTTATGGG }\end{array}$ & 508 \\
\hline TGF- $\beta 1$ & NM_000660.5 & $\begin{array}{l}\text { Forward: TATTGAGCACCTTGGGCACT } \\
\text { Reverse: ACCTCTCTGGGCTTGTTTCC }\end{array}$ & 130 \\
\hline GAPDH & NM_001289746.1 & $\begin{array}{l}\text { Forward: GAAGGTGAAGGTCGGAGTC } \\
\text { Reverse: GAAGATGGTGATGGGATTTC }\end{array}$ & 226 \\
\hline
\end{tabular}

COL, collagen; MMP, matrix metalloproteinase; TIMP, tissue inhibitor of MMP; TGF, transforming growth factor.

Primary cell culture. Human uterosacral ligamental fibroblasts (hUSLFs) were developed from fresh tissue of USL biopsies, according to Gibco/Thermo Fisher Scientific, Inc. (Waltham, MA, USA) protocols for primary culture, as previously described $(18,19)$. The cells at passages $4-6$ were used for the subsequent experiments. Recombinant human TGF- $\beta 1$ protein was obtained from PeproTech China (Suzhou, China).

Mechanical strain loading. hUSLFs were seeded on flexible plates which were pre-coated with rat tail collagen type I $(25 \mu \mathrm{g} / \mathrm{ml}$ in $0.02 \mathrm{~N}$ acetic acid; Sigma). Following reaching confluence, cells were pre-treated with recombinant human TGF- $\beta 1$ at the indicated concentrations for $24 \mathrm{~h}$, then incubated in serum-free DMEM for $12 \mathrm{~h}$ and prepared for mechanical strain. To exert cyclic mechanical stretching (CMS) on the cells, a 4-point bending device (SXG4201; Chengdu Miracle Chemicals Co., Ltd., Chengdu, China) was used $(19,20)$. The mechanical strain parameter was set at $0.3 \mathrm{~Hz}$ of $5,333 \mu \varepsilon$ (loading displacement is $4 \mathrm{~mm}$ ) for $4 \mathrm{~h}$.

Cell counting kit-8 (CCK-8) assay. Cytotoxicity was measured using a CCK-8 assay (Beyotime Biotech). According to the protocol, following treatment with recombinant human TGF- $\beta 1$ and/or CMS loading, cell samples suspended with DMEM (100 $\mu \mathrm{l}$; Gibco/Thermo Fisher Scientific, Inc.) were inoculated into a 96 -well plate $\left(5,000\right.$ cells/well) and incubated at $37^{\circ} \mathrm{C}$ for $24 \mathrm{~h}$, each sample was loaded in triplicate. Subsequently, $10 \mu \mathrm{l} \mathrm{CCK}-8$ solution were added to each well and incubated for $4 \mathrm{~h}$ at $37^{\circ} \mathrm{C}$. The absorbance was measured at $450 \mathrm{~nm}$ using a microplate spectrophotometer (Victor3-1420 Multilable Counter; PerkinElmer, Inc., Waltham, MA, USA). The viability of the treatment group was expressed as a percentage of the control group, which was designated as $100 \%$.
Gelatin zymography. A gelatin zymography assay kit (P1700; Applygen Technologies, Inc., Beijing, China) was used to examine the gelatinolytic activity of the conditioned medium. In brief, the samples ( $30 \mu \mathrm{l}$ of conditioned medium) were electrophoresed on a $10 \%$ SDS polyacrylamide gel containing $1 \mathrm{mg} / \mathrm{ml}$ gelatin under non-reducing conditions. The volume of conditioned medium loaded per lane was standardized on the basis of the cell count at harvest. To activate the proteinases, the gels were incubated at $37^{\circ} \mathrm{C}$ overnight in an incubation buffer containing $50 \mathrm{mM}$ Tris- $\mathrm{HCl}\left(\mathrm{pH} \mathrm{7.5),} 10 \mathrm{mM} \mathrm{CaCl}_{2}\right.$, and $0.02 \mathrm{mM} \mathrm{NaN}_{3}$. The gels were subsequently fixed and stained with $0.25 \%$ Coomassie brilliant blue R-250 (Maixin Biotech Co., Ltd.) for $1 \mathrm{~h}$ and washed in $25 \%$ methanol and $7 \%$ acetic acid to visualize the bands of proteolytic activity. White lysis zones indicated the degrading activity.

Reverse transcription-quantitative PCR (RT-qPCR). Total RNA was extracted using TRIzol reagent (Invitrogen, Carlsbad, CA, USA). cDNA synthesis was performed with $2 \mu \mathrm{g}$ of total RNA in a reaction volume of $20 \mu \mathrm{l}$ according to the protocol of RevertAid First Strand cDNA Synthesis kit (K1622; Fermentas, Glen Burnie, MD, USA). Quantitative PCR (qPCR) assays for relative quantification were performed using the SYBR Premix Ex Taq system (RR820A; Takara Bio, Inc., Otsu, Japan) and an ABI 7500 Fast Real-Time PCR system (Applied Biosystems Life Technologies, Foster City, CA, USA). The total reaction volume was $20 \mu \mathrm{l}$. SYBR-Green was used to amplify detection. The cycling conditions were as follows: stage $1,30 \mathrm{sec}$ at $95^{\circ} \mathrm{C}$; stage 2,40 cycles of 5 and $34 \mathrm{sec}$ at $95^{\circ} \mathrm{C}$ and $60^{\circ} \mathrm{C}$, respectively; stage $3,15 \mathrm{sec}, 1 \mathrm{~min}, 15 \mathrm{sec}$, and $15 \mathrm{sec}$ at $95^{\circ} \mathrm{C}$, $60^{\circ} \mathrm{C}, 95^{\circ} \mathrm{C}$, and $60^{\circ} \mathrm{C}$, respectively. Primers were synthesized by Sangon Biotech (Shanghai, China). Sequence data are presented in Table I. 
Table II: Demographic and clinical characteristics of all participants.

\begin{tabular}{lcccc}
\hline Parameters & POP group $(\mathrm{n}=30)$ & Control group $(\mathrm{n}=30)$ & $\mathrm{t}$-value or $\chi^{2}$-value & P-value \\
\hline Age (years, mean \pm SD) & $58.6 \pm 4.1$ & $57.1 \pm 3.3$ & $1.877^{\mathrm{a}}$ & NS \\
Parity (mean \pm SD) & $2.3 \pm 1.6$ & $2.4 \pm 1.1$ & $0.337^{\mathrm{b}}$ & NS \\
BMI (mean \pm SD) & $24.6 \pm 2.7$ & $25.2 \pm 2.4$ & $1.100^{\mathrm{a}}$ & NS \\
Post-menopausal duration & & & & NS \\
(months, means \pm SD) & $43.8 \pm 8.6$ & $40.7 \pm 7.9$ & $1.761^{\mathrm{a}}$ & .
\end{tabular}

${ }^{\mathrm{a}} \mathrm{t}$-value; ${ }^{\mathrm{b}} \chi^{2}$-value; NS, no significance (P>0.05); POP, pelvic organ prolapse; BMI, body mass index.

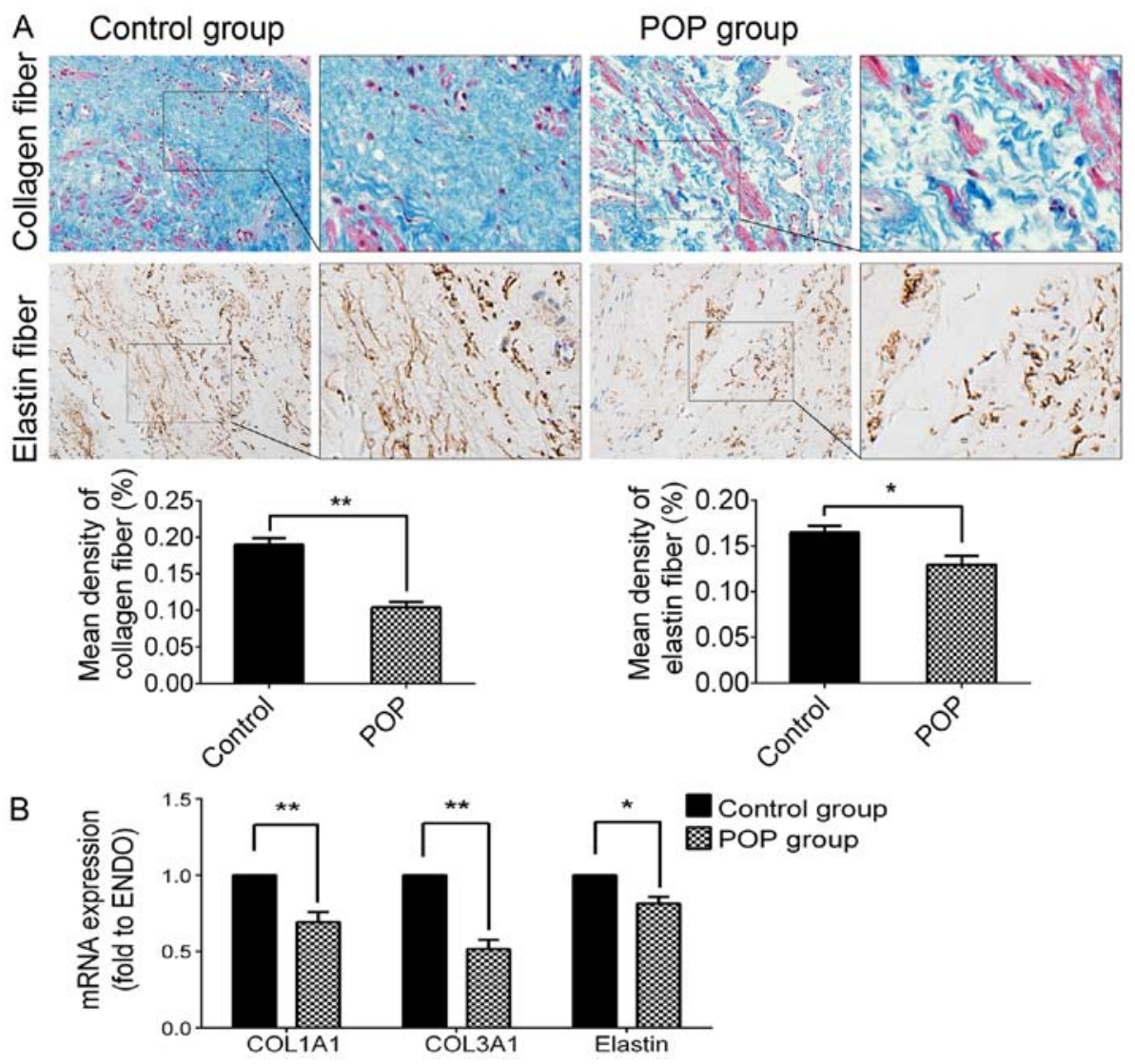

Figure 1. Expression levels of collagen fibers and elastin in USL tissue from the control group and the POP group. (A) Upper panel, by Masson's trichrome staining, collagen fibers are stained blue, smooth muscle and cytoplasm are stained red, cell nuclei are stained black; second panel, immunohistochemical staining of elastin fiber, strong positive in the control group, moderately positive in the POP group; third panel, statistical analysis based on the IOD value and mean density captured by Image-Pro plus 6.0 software. (B) The mRNA expression levels of COL1A1, COL3A1 and elastin detected by RT-qPCR. Data are presented as the means \pm standard deviation for at least 3 independent experiments. $n=30$; ${ }^{*} \mathrm{P}<0.05$ vs. the control group, ${ }^{* *} \mathrm{P}<0.01$ vs. the control group. USL, uterosacral ligament; POP, pelvic organ prolapse; COL, collagen; IOD, integrated optical density.

A preprogrammed dissociation protocol was used following amplification to ensure that all samples exhibited a single amplification. Gene expression was normalized to the housekeeping genes, GAPDH. mRNA levels were determined using the $\Delta \Delta \mathrm{Cq}$ method (Applied Biosystems Life Technologies) and expressed relative to an external calibrator on each plate.

In order to examine the clinical relevance of TGF- $\beta 1$ mRNA expression with the severity of POP, all data were re-grouped into 5 subgroups according to the grade of POP, including the non-POP $(n=20)$, POP I $(n=10)$, POP II $(n=10)$, POP III $(\mathrm{n}=10)$ and POP IV group $(\mathrm{n}=10)$.
Statistical analysis. Data are summarized as the means \pm standard deviation (SD). The Chi-square test and Student's t-test were was used to determine statistical difference between 2 groups, for numeration data and measurement data, respectively. The statistical comparison among multiple groups was processed with one-way ANOVA, and Bonferroni's multiple comparisons test was used for intergroup comparison. The detail statistical treatments and graph plotting were performed with the software of SPSS version 19.0 (IBM SPSS, Armonk, NY, USA) and GraphPad Prism version 5.01 (GraphPad Software, Inc., La Jolla, CA, USA). 


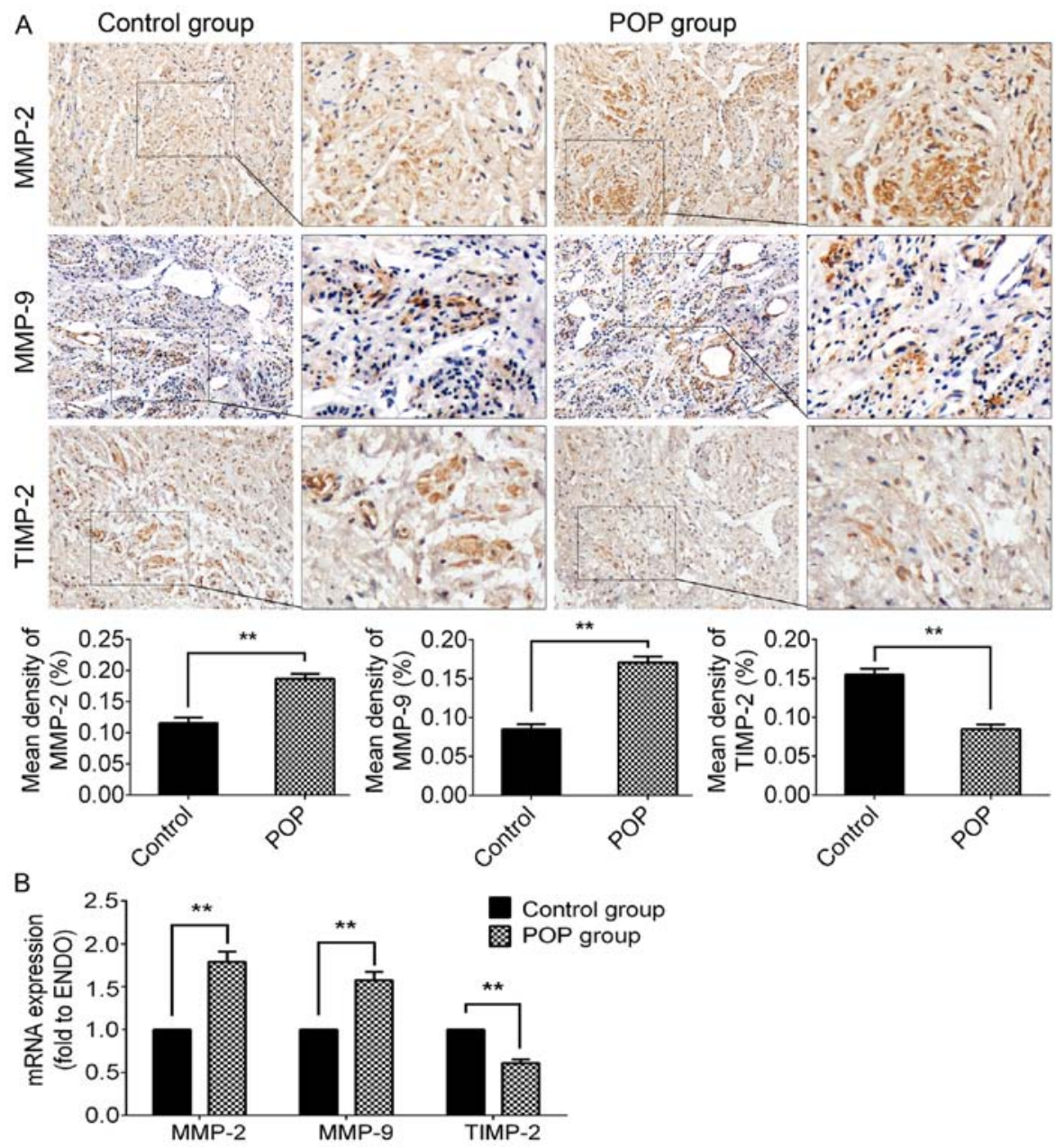

Figure 2. Expression levels of MMP-2, MMP-9 and TIMP-2 in USL tissue from the control group and the POP group. (A) Immunohistochemical staining of USL sections; top panel, for MMP-2, moderate positive staining was observed in the control group, and strong positive staining in the POP group; second panel, for MMP-9, mildly positive staining was observed in the control group, while moderately positive staining was observed in the POP group; third panel, for TIMP-2, moderately positive staining was observed in the control group, and mildly positive staining in the POP group; fourth panel, statistical comparison between the control and POP group. (B) The mRNA expression levels of MMP-2, MMP-9, and TIMP-2, examined by RT-qPCR. Data are presented as the means \pm standard deviation for at least 3 independent experiments. $n=30 ;{ }^{* *} \mathrm{P}<0.01$ vs. the control group. MMP, matrix metalloproteinase; TIMP, tissue inhibitor of MMP; USL, uterosacral ligament; POP, pelvic organ prolapse.

A value of $\mathrm{P}<0.05$ was considered to indicate a statistically significant difference.

\section{Results}

Well-matched demographic and clinical characteristics. A total of 60 women were enrolled in the present study: 30 with symptomatic POP (POP II-IV) constituted the POP group, another 30 with non-POP or asymptomatic POP (POP I) served as the control group. The participants from each group were well matched in terms of age, parity, BMI and post-menopausal duration; accordingly, there was no significant difference between the POP group and the control group $(\mathrm{P}>0.05$; Table II).

Decreased expression of collagen and elastin fibers in the $P O P$ group. In order to examine the histomorphological changes of collagen and elastin fibers in the slices of USL tissue with symptomatic POP, the methods of Masson's trichrome and IHC staining were used. As shown in Fig. 1, collagen fibers in the slices of USL tissue from the POP group were much more loose, and decreased significantly compared with the control group $(\mathrm{P}<0.05)$. Elastin fibers became shortened, condensed and cluttered in rearrangements, and the immunochemical activity of elastin in the POP group was significantly lower than that of the control group $(\mathrm{P}<0.05$; Fig. 1A). The RT-qPCR data revealed the mRNA expression levels of COL1A1 $(\mathrm{P}<0.01)$, COL3A1 $(\mathrm{P}<0.01)$ and elastin $(\mathrm{P}<0.05)$ were significantly decreased in the POP group (Fig. 1B). These results suggest that the hypo-anabolism and morphological changes of collagen and elastin fibers may be related to the pathogenesis of POP.

Upregulated expression of MMP-2/9 and downregulated expression of TIMP-2 in the POP group. The methods of IHC and RT-qPCR were used to investigate the expression of MMP-2/9 and TIMP-2 (Fig. 2). In the POP group, the immunochemical activity of either MMP-2 $(\mathrm{P}<0.01)$ or MMP-9 $(\mathrm{P}<0.01)$ was significantly stronger than that of the control group. Moreover, there was a significantly lower expression of TIMP-2 in the POP group than in the control group $(\mathrm{P}<0.01$; Fig. $2 \mathrm{~A})$. The trends in the alterations in mRNA expression were consistent with the results of IHC (Fig. 2B). The above-mentioned data 


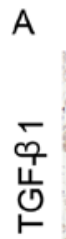
Control group POP group
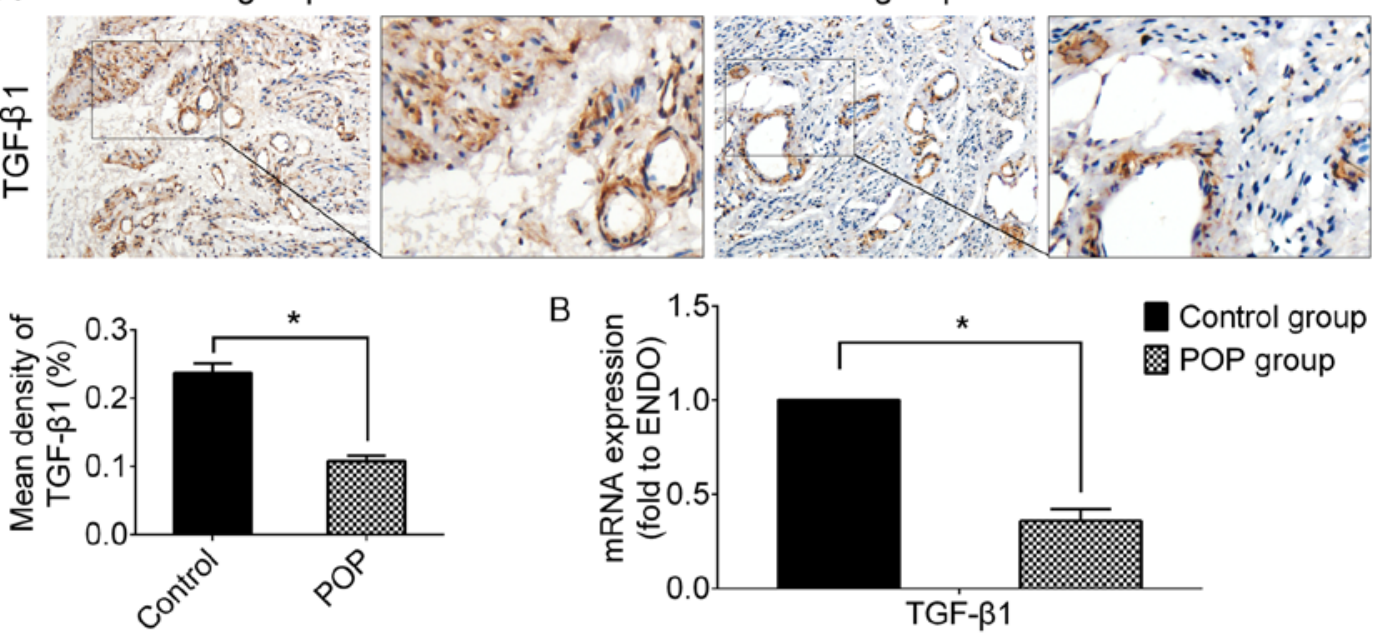

B
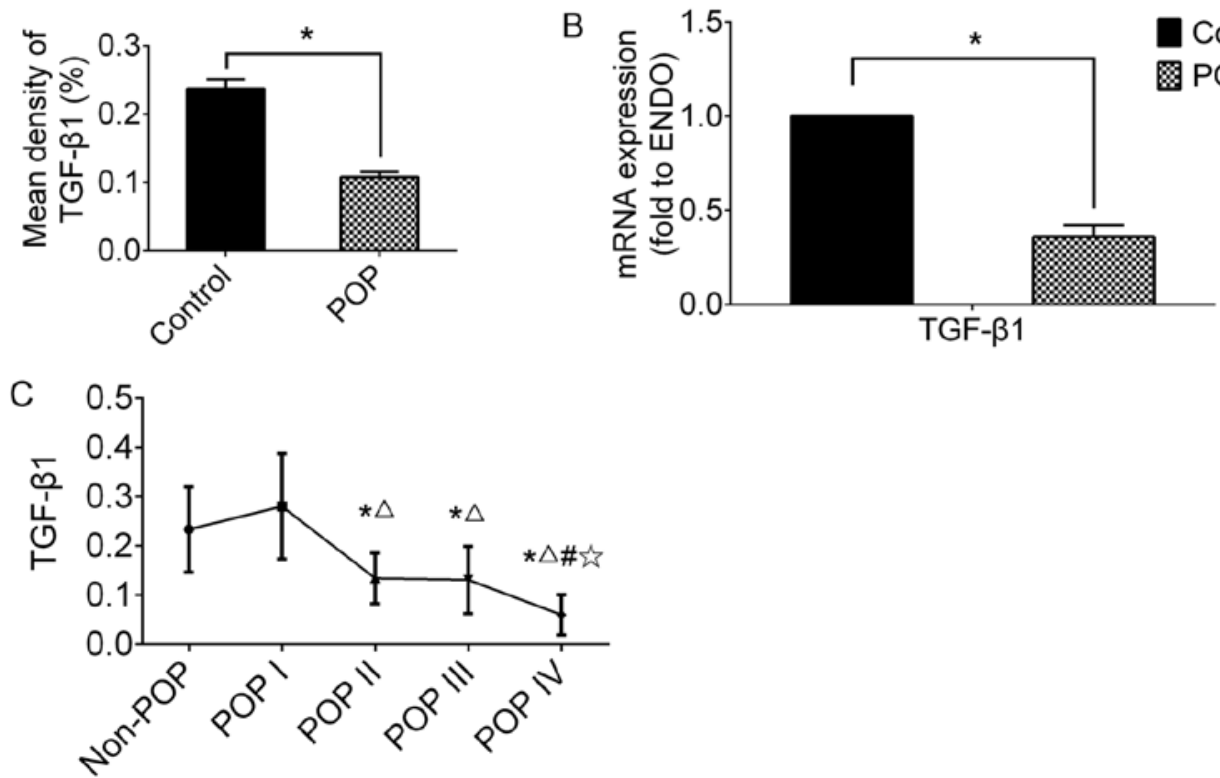

Figure 3. The expression of TGF- $\beta 1$ in USL tissue from the control group (including non-POP and asymptomatic POP of grade I) and the POP group (POP II-IV), (A) Immunohistochemical staining of USL sections; strongly positive staining was observed in the control group, and mildly positive in the POP group; $\mathrm{n}=30$; (B) the mRNA expression levels of TGF- $\beta 1$ examined by RT-qPCR. " $\mathrm{P}<0.05$ vs. the control group. $n=30$; (C) analyzing the clinical relevance of TGF- $\beta 1 \mathrm{mRNA}$ expression level with the grade of POP. Data are presented as the means \pm standard deviation for at least 3 independent experiments; $n=10 ;{ }^{*},{ }^{\Delta,},{ }^{*}$ and ${ }^{\not *}$ represent $\mathrm{P}<0.05$ when compared with the non-POP, POP I, POP II, and POP III group, respectively. TGF, transforming growth factor; USL, uterosacral ligament; POP, pelvic organ prolapse.

suggest that the increased degradation of ECM proteins may be involved in the pathogenesis of POP.

Expression level of TGF- $\beta 1$ has clinical relevance with the severity of POP. IHC and RT-qPCR were performed using USL tissue to examine the expression of TGF- $\beta 1$ (Fig. 3). In the POP group, the expression of TGF- $\beta 1$ was significantly lower than that of the control group, as shown by both IHC and RT-qPCR $(n=30, P<0.05)$.

In order to examine the clinical relevance of TGF- $\beta 1$ mRNA expression with the severity of POP, all data were re-grouped into 5 subgroups according to the grade of POP, including the non-POP ( $n=20)$, POP I $(n=10)$, POP II $(n=10)$, POP III $(n=10)$ and POP IV group $(n=10)$. The results revealed that the mRNA expression level of TGF- $\beta 1$ in the POP group (POP II-IV) was significantly lower than that of the control group (non-POP or POP I). Additionally, Bonferroni's multiple comparisons test among the subgroups from POP I to POP IV was carried out to determine the correlation between TGF- $\beta 1$ and the severity of disease. The results revealed a tendency for a negative correlation between the mRNA expression of TGF- $\beta 1$ and the grade of POP in a severity-dependent manner; however, there was no significant difference between the non-POP and POP I subgroups (Fig. 3C).
TGF- $\beta 1$ attenuates the CMS-induced degradation of ECM proteins in a cell model established using hUSLFs in vitro. To determine whether TGF- $\beta 1$ plays a role in ECM remodeling generated by hUSLFs in the pathogenesis of POP, we replicated the cell model induced by CMS with certain parameters as described in our previous study $(19,20)$. In cell experiments in the present study, hUSLFs at passages 4-6 were pre-treated with recombinant human TGF- $\beta 1$ at concentrations of 0,5 , or $10 \mathrm{ng} / \mathrm{ml}$ for $24 \mathrm{~h}$, and then exposed to CMS with a strain of 5,333 $\mu \varepsilon$ for 4 h. As shown in Fig. 4, pre-treatment with TGF- $\beta 1$ significantly attenauted the decrease in cell viability induced by CMS. In the absence of TGF- $\beta 1$, CMS not only reduced the synthesis of COL1A1 $(\mathrm{P}<0.05)$, COL3A1 $(\mathrm{P}<0.05)$ and elastin $(\mathrm{P}<0.05)$, but also promoted their degradation through the inhibition of the expression of TIMP-2 $(\mathrm{P}<0.05)$ and the promotion of the expression of MMP-2/9 $(\mathrm{P}<0.05)$. In addition, pre-treatment with TGF- $\beta 1$ significantly attenuated the CMS-induced degradation of COL1A1 and elastin by inhibiting the proteolytic activities of MMP-2/9 $(\mathrm{P}<0.05)$. The above-mentioned data indicate that TGF- $\beta 1$ treatment plays a therapeutic role in the CMS-induced excessive degradation of the ECM.

Phosphorylation of the Smad3 signaling pathway is significantly improved by pre-treatment with TGF- $\beta 1$ 
A
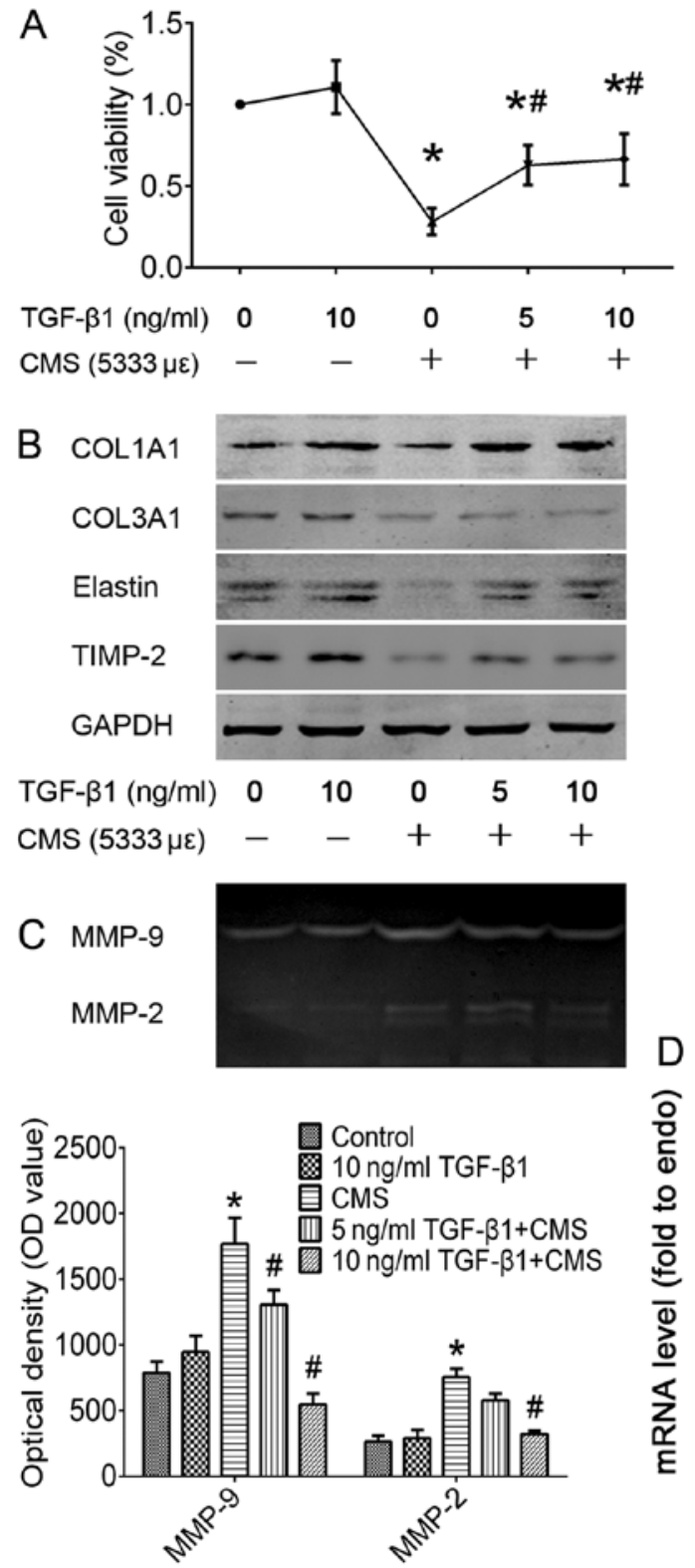

COL1A1

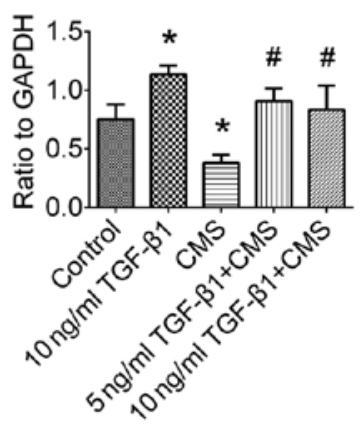

Elastin

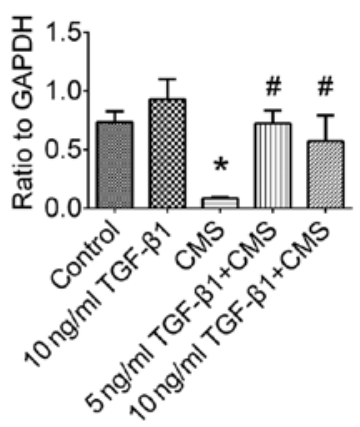

COL3A1

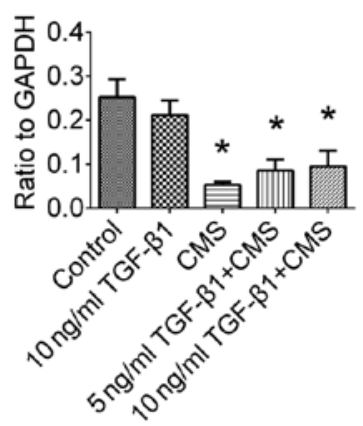

TIMP-2

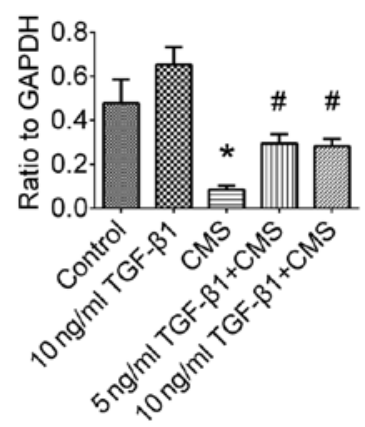

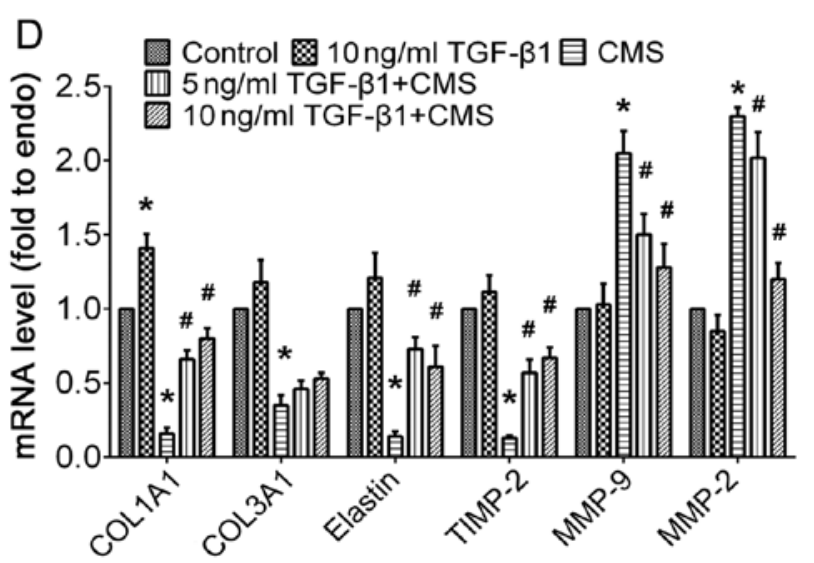

Figure 4. Effect of TGF- $\beta 1$ on cell viability and ECM metabolism in the control or hUSLFs subjected to CMS. In the study groups, hUSLFs were incubated with recombinant human TGF- 1 at concentrations of 0,5 , or $10 \mathrm{ng} / \mathrm{ml}$ for $24 \mathrm{~h}$, then exposed to CMS at a strain of 5,333 $\mu \varepsilon$ for $4 \mathrm{~h}$. The normal control group was not treated with TGF- $\beta 1$ or CMS. (A) Cell viability was detected using the Cell Counting kit-8 (CCK-8) method. Expression levels of COL1A1, COL3A1, elastin, TIMP-2, MMP-9 and MMP-2 were analyzed by (B) western blot analysis, (C) gelatin zymography, or (D) RT-qPCR. Data represent the means \pm standard deviation for at least 3 independent experiments; $\mathrm{n}=3 ;{ }^{*} \mathrm{P}<0.05$ vs. the control group; ${ }^{\#} \mathrm{P}<0.05$ vs. the CMS group. TGF, transforming growth factor ECM, extracellular matrix; CMS, cyclic mechanical stretching; hUSLF, human USL fibroblast; COL, collagen; TIMP, tissue inhibitor of MMP; MMP, matrix metallloproteinase.

following CMS. The TGF- $\beta 1 /$ Smad signaling pathway plays an important role in fibrosis and degenerative fibrotic disease. In order to identify the exact regulatory mechanisms through which TGF- $\beta 1$ attenuates the ECM remodeling induced by CMS, the alterations in downstream Smad signaling pathways were investigated by western blot analysis (Fig. 5). In the present study, it was observed that the phosphorylation rates of both Smad 2 and 3 were significantly inactivated by CMS in the hUSLFs $(\mathrm{P}<0.05)$. Pre-treatment with TGF- $\beta 1$ significantly improved the phosphorylation rate of Smad3 $(\mathrm{P}<0.05)$, but had no significant effect on the phosphorylation of $\operatorname{Smad} 2(\mathrm{P}>0.05)$. In addition, western blot analysis revealed that the Smad4 signaling pathway exhibited no significant alteration induced following CMS ( $\mathrm{P}>0.05)$. It was thus suggested that the Smad3 signaling pathway is the downstream regulatory mechanism activated by TGF- $\beta 1$ in hUSLFs subjected to CMS.

\section{Discussion}

POP is currently considered to be a disorder of pelvic floor dysfunction, which is caused by the degenerative weakening of pelvic supportive structures (21). Based on previous studies, collagen and elastin, as the essential components of connective tissue, contribute the most to the maintenance of resistance ability (6,22). Although the histopathological alterations in connective tissue in POP women have been mentioned previously $(22,23)$, there are disputes as to the trends in the alterations of the levels of collagen I, III and elastin. 


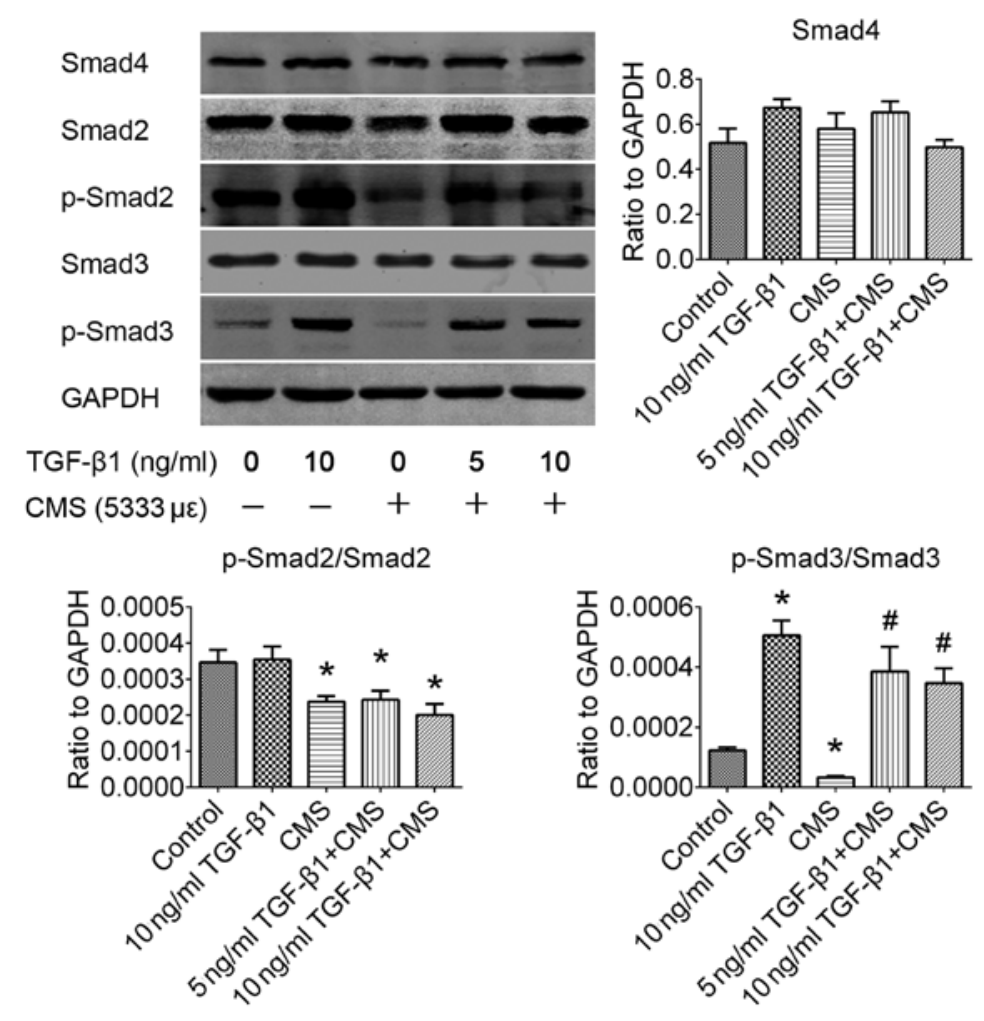

Figure 5. Involvement of the TGF- $\beta 1 /$ Smad3 signaling pathway in the regulatory mechanism of ECM remodeling in hUSLFs subjected to CMS. Western blot analysis validated that pre-treatment with TGF- $\beta 1$ significantly promoted the phosphorylation of the Smad 3 signaling pathway in the cells subjected to CMS Data represent the means \pm standard deviation for at least 3 independent experiments; $n=3$; ${ }^{*} \mathrm{P}<0.05$ vs. the control group; ${ }^{\text {}} \mathrm{P}<0.05$ vs. the CMS group. ECM, extracellular matrix; CMS, cyclic mechanical stretching; hUSLF, human USL fibroblast; TGF, transforming growth factor.

Vulic et al (24) and Chen et al (25) reported that the amounts of collagen I and III were significantly decreased in USL with POP. However, there are different opinions, as Yucel et al (26) clarified that the expression of collagen III was upregulated in women with POP. Gabriel et al (8) concluded that there was no significant difference in the expression of collagen I between women with POP and or women with non-POP, but an increased ratio of collagen I/III may be a salient feature of POP. Methodological issues may account for these discrepancies. A common feature in the above-mentioned results is that most of these experimental data were obtained from a single IHC investigation; such evidence would inevitably have limitations and one-sidedness, as IHC data are always limited by the biopsy site and sample size. Different biopsy sites, particularly those in prolapsed tissue, also increase variability as the differences in stress loads can upregulate different proteins. Additionally, pathological diagnosis is susceptible to the subjective judgement of the pathologist. To a certain extent, RT-qPCR data is more objective. In order to obtain more objective experimental data, in addition to IHC, we also carried out RT-qPCR as a further complement. In the present study, our experimental data validated that the expression levels of COL1A1, COL3A1 and elastin were significantly decreased in USL tissue from the POP group compared with the control group (Fig. 1). It can be inferred that the loss of collagen and elastin is associated with the development of POP.

MMPs and TIMPs are considered key regulators in the degradation of ECM, which are commonly involved in physiological and pathological proteolytic processes. A previous study found a lower amount of collagen, and higher expression levels of MMP-2/9 in vaginal biopsies from women with POP (14). A significantly higher immunoreactivity in MMP-1, but no difference in MMP-2, was observed in women with POP compared with the controls $(24,27)$. Gabriel et al (28) reported that an increased MMP-2 expression in USL correlated with the incidence of POP. Dviri et al (29) found higher expression levels of MMP-1/9 in vaginal wall biopsies from women with POP compared with the controls. To analyze the reasons for discrepancies in the above-mentioned findings, firstly, the effects of confounding factors, such as age, parity, obesity, menopausal status and a history of HRT should be considered. Secondly, differences in research methods may lead to divergent conclusions. Compared with western blot analysis, gelatin zymography, immunochemistry andRT-qPCR are more suitable for the study of MMP expression, as gelatin zymography directly exhibits the activity of proteolytic enzymes, immunochemistry additionally shows the distribution of MMPs, and RT-qPCR reflects changes at the transcriptional level, while western blot analysis can only reflect the protein content of MMPs. In the present study, the method of either immunochemistry or gelatin zymography combined with RT-qPCR was selected to study MMPs. A significantly higher expression of MMP-2/9, as well as a lower expression of TIMP-2, was observed in USL from women with POP compared with the asymptomatic controls after being matched in age, parity, BMI and menopausal duration (Fig. 2). The present results are highly in accordance with those of previous reports $(30,31)$. According to the experimental results, 
we suggest not only a reduced synthesis, but also an increased degradation of ECM, which are the metabolic abnormalities which contribute to the incidence of POP.

TGF- $\beta$ is currently considered as an important regulator widely involved in the pathogenesis of fibrosis and degenerative fibrotic diseases, that may induce fibroblast differentiation, promote collagen synthesis and reduce degradation by inhibiting MMPs and upregulating TIMPs (12). Previous researchers have clarified that the activity of gelatinase/type IV collagenase (MMP-2) was regulated by TGF- $\beta 1$ in human gingival fibroblasts (32). TGF- $\beta 1$ reduced the level of collagenase mRNA and increased the level of TIMP mRNA. A sparse, but growing evidence of TGF- $\beta 1$ modulation in pelvic connective tissue has been found. The TGF- $\beta /$ Smad signaling pathway and MMPs are involved in stress urinary incontinence induced by vaginal delivery in animal studies (15). However, to the best of our knowledge, TGF- $\beta 1$ has rarely been investigated in women with POP. In the present study, TGF- $\beta 1$ was found to be expressed at significantly lower levels in USL tissue from women with POP than in the controls, and further statistical analysis after re-grouping according to the grade of POP indicated the mRNA expression level of TGF- $\beta 1$ partially negatively correlated with the severity of POP (Fig. 3). Perhaps more statistically significant results may have been obtained if a greater number of participants were enrolled in this study. Taken together with the published theory in the relative field, at least we could predict the possible involvement of TGF- $\beta 1$ in the pathogenesis and development of POP by regulating the metabolism of ECM.

To identify the exact role of TGF- $\beta 1$ in the development of POP, in the follow-up experiment in vitro, we introduced a cellular disease model that mimicked ECM remodeling in POP, which was established on hUSLFs subjected to CMS loading with a strain of $5,333 \mu \varepsilon$ for $4 \mathrm{~h}$ as described in our previous study $(19,20)$. Since it has been conducted in a previous study $(19,20)$, we did not describe the dose-effect investigation in detail in this study. To verify the rationality of the cell model, it was observed that CMS loading not only significantly stimulated the degradation of ECM proteins, but also reduced its synthesis (Fig. 4); these effects are metabolic features in POP. In vitro cell experimental results indicated that pre-treatment with TGF- $\beta 1$ significantly attenuated the CMS-induced loss of ECM proteins by inhibiting the proteolytic activities of MMP-2/9, as well as by stimulating synthesis of COL1A1 and elastin. Further experiments revealed that the phosphorylation of the Smad3 signaling pathway is the downstream regulatory mechanism activated by TGF- $\beta 1$ in hUSLFs subjected to CMS loading (Fig. 5).

There are still some shortcomings to the present study. Firstly, we used a relatively small sample size. With a larger patient number, perhaps more statistically significant results may have been obtained following re-grouping according to the grade of POP. Secondly, IHC is not the most ideal method for the study of the expression of MMPs at the tissue level, since the secretion and activity of MMPs may always change quantitatively, and in theory, gelatin zymography is the more suitable method. However, due to the limited source of clinical specimens and the experimental budget, we selected IHC as the study method, as IHC has a certain advantage in detecting the localization of MMP expression in tissue to a certain extent. Thirdly, due to time and experimental technology constraints, the present study was limited, resulting in the lack of adequate evidence to confirm whether the Smad3 signaling pathway is the only downstream regulatory mechanism. In addition, the present conclusion may be more convincing if further validation was available at the animal level experiment in vivo.

In conclusion, in the present study, we provide evidence to indicate that the reduced anabolism and increased catabolism of ECM proteins in USL are the pathological characteristics of POP. TGF- $\beta 1$ not only can be used as a biomarker to predict the severity of POP, but also has a therapeutic effect in cell model of POP, and it is suggested that the TGF- $\beta 1 / \mathrm{Smad} 3$ signaling pathway may be a novel therapeutic target for POP.

\section{Acknowledgements}

This study was funded by the National Natural Science Foundation of China (NSFC) (grant no. 81270684) and the Special Fund for Basic Scientific Research in National central Colleges and Universities (grant no. 2042017kf0114). We would like to thank Professor Xu Xue-Xian, Ms. Li Yan-Bo, Ms. Cheng Yan-Xiang, Mr. Luo Ruo-Yu, at the Department of Gynecology and Obstetrics, Renmin Hospital of Wuhan University for assisting with the specimen biopsies.

\section{References}

1. Barber MD and Maher C: Epidemiology and outcome assessment of pelvic organ prolapse. Int Urogynecol J Pelvic Floor Dysfunct 24: 1783-1790, 2013.

2. Olsen AL, Smith VJ, Bergstrom JO, Colling JC and Clark AL: Epidemiology of surgically managed pelvic organ prolapse and urinary incontinence. Obstet Gynecol 89: 501-506, 1997.

3. Subak LL, Waetjen LE, van den Eeden S, Thom DH, Vittinghoff E and Brown JS: Cost of pelvic organ prolapse surgery in the United States. Obstet Gynecol 98: 646-651, 2001.

4. Merrill RM: Hysterectomy surveillance in the United States, 1997 through 2005. Med Sci Monit 14: CR24-CR31, 2008.

5. De Landsheere L, Munaut C, Nusgens B, Maillard C, Rubod C, Nisolle M, Cosson M and Foidart JM: Histology of the vaginal wall in women with pelvic organ prolapse: A literature review. Int Urogynecol J Pelvic Floor Dysfunct 24: 2011-2020, 2013.

6. Chen B and Yeh J: Alterations in connective tissue metabolism in stress incontinence and prolapse. J Urol 186: 1768-1772, 2011.

7. Cole EE, Leu PB, Gomelsky A, Revelo P, Shappell H, Scarpero HM and Dmochowski RR: Histopathological evaluation of the uterosacral ligament: Is this a dependable structure for pelvic reconstruction? BJU Int 97: 345-348, 2006.

8. Gabriel B, Denschlag D, Göbel H, Fittkow C, Werner M, Gitsch G and Watermann D: Uterosacral ligament in postmenopausal women with or without pelvic organ prolapse. Int Urogynecol J Pelvic Floor Dysfunct 16: 475-479, 2005.

9. Goh JT: Biomechanical and biochemical assessments for pelvic organ prolapse. Curr Opin Obstet Gynecol 15: 391-394, 2003.

10. Nagase H, Visse R and Murphy G: Structure and function of matrix metalloproteinases and TIMPs. Cardiovasc Res 69: 562-573, 2006.

11. Ra HJ and Parks WC: Control of matrix metalloproteinase catalytic activity. Matrix Biol 26: 587-596, 2007.

12. Sampson N, Berger P and Zenzmaier C: Redox signaling as a therapeutic target to inhibit myofibroblast activation in degenerative fibrotic disease. BioMed Res Int 2014: 131737, 2014.

13. Mosier E, Lin VK and Zimmern P: Extracellular matrix expression of human prolapsed vaginal wall. Neurourol Urodyn 29: 582-586, 2010.

14. Budatha M, Roshanravan S, Zheng Q, Weislander C, Chapman SL, Davis EC, Starcher B, Word RA and Yanagisawa H: Extracellular matrix proteases contribute to progression of pelvic organ prolapse in mice and humans. J Clin Invest 121: 2048-2059, 2011 
15. Klutke J, Ji Q, Campeau J, Starcher B, Felix JC, Stanczyk FZ and Klutke C: Decreased endopelvic fascia elastin content in uterine prolapse. Acta Obstet Gynecol Scand 87: 111-115, 2008.

16. Li BS, Hong L, Min J, Wu DB, Hu M and Guo WJ: The expression of glutathione peroxidase-1 and the anabolism of collagen regulation pathway transforming growth factor-beta1-connective tissue growth factor in women with uterine prolapse and the clinic significance. Clin Exp Obstet Gynecol 40: 586-590, 2013.

17. Bump RC, Mattiasson A, Bø K, Brubaker LP, DeLancey JO, Klarskov P, Shull BL and Smith AR: The standardization of terminology of female pelvic organ prolapse and pelvic floor dysfunction. Am J Obstet Gynecol 175: 10-17, 1996.

18. Liu C, Yang Q, Fang G, Li BS, Wu DB, Guo WJ, Hong SS and Hong L: Collagen metabolic disorder induced by oxidative stress in human uterosacral ligament derived fibroblasts: A possible pathophysiological mechanism in pelvic organ prolapse. Mol Med Rep 13: 2999-3008, 2016.

19. Li BS, Guo WJ, Hong L, Liu YD, Liu C, Hong SS, Wu DB and Min J: Role of mechanical strain-activated PI3K/Akt signaling pathway in pelvic organ prolapse. Mol Med Rep 14: 243-253, 2016.

20. Hong S, Li H, Wu D, Li B, Liu C, Guo W, Min J, Hu M, Zhao Y and Yang Q: Oxidative damage to human parametrial ligament fibroblasts induced by mechanical stress. Mol Med Rep 12: 5342-5348, 2015.

21. Jelovsek JE, Maher C and Barber MD: Pelvic organ prolapse. Lancet 369: 1027-1038, 2007.

22. Moon YJ, Choi JR, Jeon MJ, Kim SK and Bai SW: Alteration of elastin metabolism in women with pelvic organ prolapse. J Urol 185: 1786-1792, 2011.

23. Han L, Wang L, Wang Q, Li H and Zang H: Association between pelvic organ prolapse and stress urinary incontinence with collagen. Exp Ther Med 7: 1337-1341, 2014.

24. Vulic M, Strinic T, Tomic S, Capkun V, Jakus IA and Ivica S: Difference in expression of collagen type I and matrix metalloproteinase-1 in uterosacral ligaments of women with and without pelvic organ prolapse. Eur J Obstet Gynecol Reprod Biol 155: 225-228, 2011
25. Chen HY, Lu Y, Qi Y, Bai WP and Liao QP: Relationship between the expressions of mitofusin-2 and procollagen in uterosacral ligament fibroblasts of postmenopausal patients with pelvic organ prolapse. Eur J Obstet Gynecol Reprod Biol 174: 141-145, 2014.

26. Yucel N, Usta A, Guzin K, Kanter M, Bilgic E, Ozel NO and Ozgul M: Immunohistochemical analysis of connective tissue in patients with pelvic organ prolapse. J Mol Histol 44: 97-102, 2013.

27. Strinic T, Vulic M, Tomic S, Capkun V, Stipic I and Alujevic I: Matrix metalloproteinases-1, -2 expression in uterosacral ligaments from women with pelvic organ prolapse. Maturitas 64: $132-135,2009$.

28. Gabriel B, Watermann D, Hancke K, Gitsch G, Werner M, Tempfer $C$ and zur Hausen $A$ : Increased expression of matrix metalloproteinase 2 in uterosacral ligaments is associated with pelvic organ prolapse. Int Urogynecol J Pelvic Floor Dysfunct 17: 478-482, 2006

29. Dviri M, Leron E, Dreiher J, Mazor M and Shaco-Levy R: Increased matrix metalloproteinases-1,-9 in the uterosacral ligaments and vaginal tissue from women with pelvic organ prolapse. Eur J Obstet Gynecol Reprod Biol 156: 113-117, 2011.

30. Alarab M, Kufaishi H, Lye S, Drutz H and Shynlova O Expression of extracellular matrix-remodeling proteins is altered in vaginal tissue of premenopausal women with severe pelvic organ prolapse. Reprod Sci 21: 704-715, 2014.

31. Liang CC, Huang HY and Chang SD: Gene expression and immunoreactivity of elastolytic enzymes in the uterosacral ligaments from women with uterine prolapse. Reprod Sci 19: 354-359, 2012.

32. Overall CM, Wrana JL and Sodek J: Transcriptional and post-transcriptional regulation of $72-\mathrm{kDa}$ gelatinase/type IV collagenase by transforming growth factor-beta 1 in human fibroblasts. Comparisons with collagenase and tissue inhibitor of matrix metalloproteinase gene expression. J Biol Chem 266: 14064-14071, 1991. 